PION NUCLEON INTERACTION AT LOW ENERGY

\author{
Manoj K. Banerjee \\ Department of Physics and Astronomy \\ University of Maryland, College Park, Maryland 20742 \\ March 1979
}

U. of Md. PT \#79-066
U. of Md. TR \# $199-076$
U. of Md. PP $\# 79-165$

MISTER

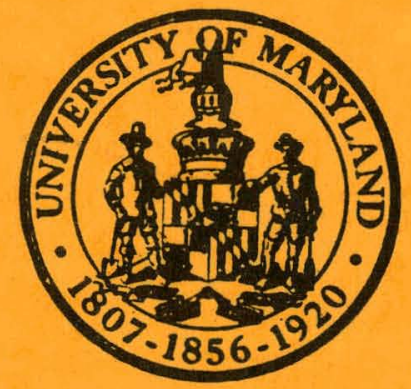

UNIVERSITY OF MARYLAND

DEPARTMENT OF PHYSICS AND ASTRONOMY COLLEGE PARK, MARYLAND

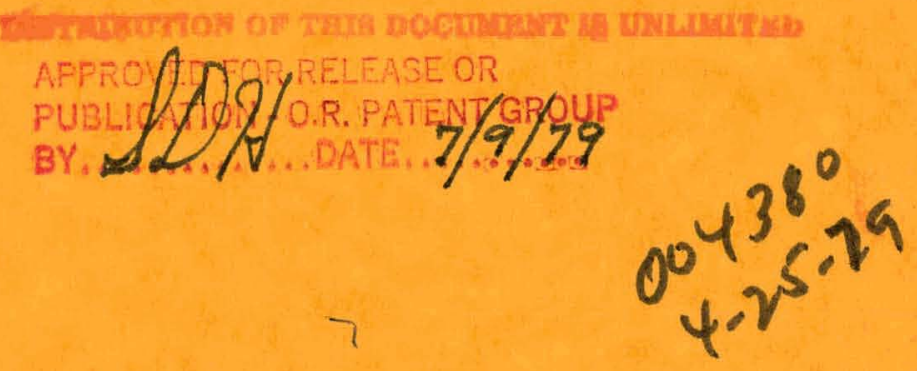




\section{DISCLAIMER}

This report was prepared as an account of work sponsored by an agency of the United States Government. Neither the United States Government nor any agency Thereof, nor any of their employees, makes any warranty, express or implied, or assumes any legal liability or responsibility for the accuracy, completeness, or usefulness of any information, apparatus, product, or process disclosed, or represents that its use would not infringe privately owned rights. Reference herein to any specific commercial product, process, or service by trade name, trademark, manufacturer, or otherwise does not necessarily constitute or imply its endorsement, recommendation, or favoring by the United States Government or any agency thereof. The views and opinions of authors expressed herein do not necessarily state or reflect those of the United States Government or any agency thereof. 


\section{DISCLAIMER}

Portions of this document may be illegible in electronic image products. Images are produced from the best available original document. 
Invited talk presented at the 2nd Int1. Conf. on Meson-Nuclear Physics, Houston, Texas, March 5-9, 1979.

ORO 5126-60

U. of Md. PT \#79-066

U. of Md. TR $\$$ F9-076

U. of Md. PP \#79-165

\title{
PION NUCLEON INTERACTION AT LOW ENERGY ${ }^{\dagger}$
}

\author{
Manoj K. Banerjee \\ Department of Physics and Astronomy \\ Unfversity of Maryland, College Park, Maryland 20742
}

The period from the mld 1950's to the late 1960's saw intensive developments in the field of $\pi N$ interaction. The classic work of Chew and Low ${ }^{1}$ was followed by developments in dispersion theories, ${ }^{2}$ current algebras, PCAC, Chiral symetry, 3 etc. During the last six or seven years there has been a resurgence of interest in $\pi \mathrm{N}$ interactions at low energies inspired entirely by the growth of vartous pion experimental facilities which have provided increasingly precise data on pion nucleus scattering and reactions. To understand the interaction of a pion with a nucleus we require the knowledge of $\pi \mathrm{N}$ scattering amplitudes for $\pi$ and $N$ 4-momenta which are off the mass shell, but real. These amplitudes can be determined only with the help of a dynamical theory. It should be noted that the usual dispersion theories deal with amplitudes where the 4-momenta are on the mass shell and, in general, complex. Since the work of Chew and Low there has not been a great deal of work in particle physics attempting to explain the low energy $\pi \mathrm{N}$ scattering data in terms of a dynamical theory. So when the need arose the intermediate energy theorists had to take up the task.

In the literature there are several papers which deal with the $S$ and $P$ wave $\pi N$ scattering amplitudes in separable form. Some of these use separable potentials; ${ }^{4}$ others are extensions of the static theory of Chew and Low. 5

In this talk $I$ will/describe a theory of $\pi N$ interaction at low energy/developed by Barry Cammarata and myself. An early version of the theory was presented at the First Meson Nucleus Conference at Carnegie-Mellon University. 6

I will try to convey the spirit of our approach by analogy with an unusual approach to potential scattering problem. Let $t$ and $y$ be the kinetic and potential energy operators, respectively. Let $|p\rangle$, $\mid \vec{q}>$ be plane wave states and $\mid \psi(+)(n)>$ be a scattering. eigenfunction of $\mathrm{H}=\mathrm{t}+\mathrm{V}$ with eigenvalue $\mathrm{E}_{\mathrm{n}}$. The half-off-shell scattering amplitude $\left\langle n\left|T\left(E_{n}\right)\right| \vec{p}\right\rangle=\left\langle\psi{ }^{(+)}(n)|v| \vec{p}\right\rangle$ satisfiess the Low equation

$$
\left\langle\vec{k}\left|T\left(E_{k}\right)\right| \vec{p}\right\rangle=\langle\vec{k}|v| \vec{p}\rangle+\sum_{n} \frac{\left\langle n\left|T\left(E_{n}\right)\right| \vec{k}\right\rangle^{*}\left\langle n\left|T\left(E_{n}\right)\right| \vec{p}\right\rangle}{E_{k}-F_{n}+i n} .
$$

If one knew all the potential matrix elements $\langle\vec{k}|v| \vec{p}\rangle$ one could consider solving the nonlinear integral equation. In a field theoretic Low expansion of the scattering amplitude the so-called seagull terms play the role of potential matrix elements. If we assume a Lagrangian we can write down, in principle, the form of the seagull terms with a few unknown parameters, such as coupling constants, form factor masses, etc. But there are serious practical problems. For

\footnotetext{
Work supported by the U. S. Department of Energy.
} 
example, consider a Lagrangian involving nucleons, a scalar-isoscalar meson ( $\sigma$ meson) and pions with pseudoscalar coupling. We are all aware of the pair suppression mechanism in the 1soscalar amplitude where the huge repulsive contribution of $-\frac{g_{\pi}^{2}}{M}$ from the $Z$-graphs is almost completely cancelled by $\frac{g_{\sigma N N} g_{\sigma \pi \pi}}{m_{\sigma}^{2}}$, the attractive contribution from the $\sigma$ meson exchange. The remainder, presumably of the same order as the physical amplitude, is about $\frac{1}{100}$ th of $\frac{g_{\pi}^{2}}{M}$. It will be very difficult for any practical calculation to reproduce this kind of result. We avoid these dangers by taking a different path. Suppose we have a way of knowing all elements of a fully offshell $\mathrm{T}$ matrix, $\left\langle\vec{k}\left|\mathrm{~T}\left(\mathrm{E}_{\mathrm{o}}\right)\right| \overrightarrow{\mathrm{p}}\right\rangle$ for some value of $\mathrm{E}_{\mathrm{o}}$ which may be quite unphysical. Then not only can we write down an integral equation $\left\langle\vec{k}\left|T\left(E_{k}\right)\right| \vec{p}\right\rangle=\left\langle k\left|T\left(E_{o}\right)\right| \vec{p}\right\rangle$

$+\sum_{n}\left\langle\vec{k}\left|T^{\dagger}\left(E_{n}\right)\right| n>\left\langle n\left|T\left(E_{n}\right)\right| \vec{p}\right\rangle\left\{\frac{1}{E_{k}-E_{n}+i n}-\frac{1}{E_{0}-E_{n}+i n}\right\}\right.$

without any reference to the potential matrix elements but we gain the additional advantage that the equation is once-subtracted, thus more rapidly convergent.

In our theory ${ }^{7}$ a soft pion limit plays the role of $T\left(E_{o}\right)$. This requires two conjectures.

Conjecture 1: Strong PCAC.

$$
\phi_{\alpha}=\frac{\sqrt{2}}{f_{\pi}} \frac{\partial A_{\mu}^{\alpha}}{\partial x_{\mu}}
$$

with $\mathrm{f}_{\pi} \equiv \dot{U} . \dot{9} 39 \mathrm{~m}_{\pi}^{3}$, is the canonical plon fièld.

Conjecture 2: Notion of $\sigma$ commutator.

$$
1 \int d^{4} y \delta\left(x_{0}-y_{o}\right)\left[A_{0}^{\alpha}(x), \partial^{\mu} A_{\mu}^{\beta}(y)\right]=\delta_{\alpha \beta} \sigma(x) \text {. }
$$

The locality of $\sigma(x)$ follows from microcausality. The statement that it is an isoscalar field is the conjecture.

It is very important to note that the foregoing conjecture implies that

$$
\int d^{4} y \delta\left(x_{0}-y_{0}\right)\left[A_{0}^{\alpha}(x), A_{\mu}^{\beta}(y)\right]
$$

is a conserved vector isovector current. We may assume, if we wish, that thic is proportional to the vector current, i.c.,

$$
\int d^{4} y \delta\left(x_{0}-y_{0}\right)\left[A_{0}^{\alpha}(x), A_{\mu}^{\beta}(y)\right]=\zeta\left\{i \varepsilon_{\alpha \beta \lambda} v_{\mu}^{\lambda}(x)\right\} .
$$

But our conjecture says nothing about the constant $\zeta$, which in current algebra equals one. Thus the notion of $\sigma$ commutator is compatible with current algebra but does not imply it completely. We do not assume current algebra and, therefore, we can use it as an external test of our theory.

When the notion of PCAC was first introduced a popular accompanying assumption was that the matrix elements of the axial vector current or of its divergence are slowly varying functions of the relevant momentum variables. We should emphasize that we make 
no such assumption.

The soft pion limit can be used to eliminate not all the seagull terms, but only the isosclar part. So a conjecture about the isovector part must be made.

Conjecture $3:^{-}$The seagull terms are purely isocalar.

The third conjecture does not allow a term in the underlying Lagranglan involving the coupling of a vector-isovector field [ $\rho$ meson] to the hadronic isospin current. Thus the $\rho$ meson must appear as a two pion resonance and not as a canonical field. In other words, we cannot draw a Feynman diagram with a $\rho \cdot m e s o n$ exchange.

While in our approach it is not necessary to spell out what the underlying Lagrangian is we may note that the Gell-Mann-Levy $\sigma$ mode 8 is completely compatible with our conjectures, and, as we shall see shortly, with some of our results. Several years back Basdevant and Lee 9 had shown how the $\rho$ and the $f$ resonances appear from the Gell-Mann - Levy o model.

Finally, our theory gives an integral equation which is shown below pictorially. 10

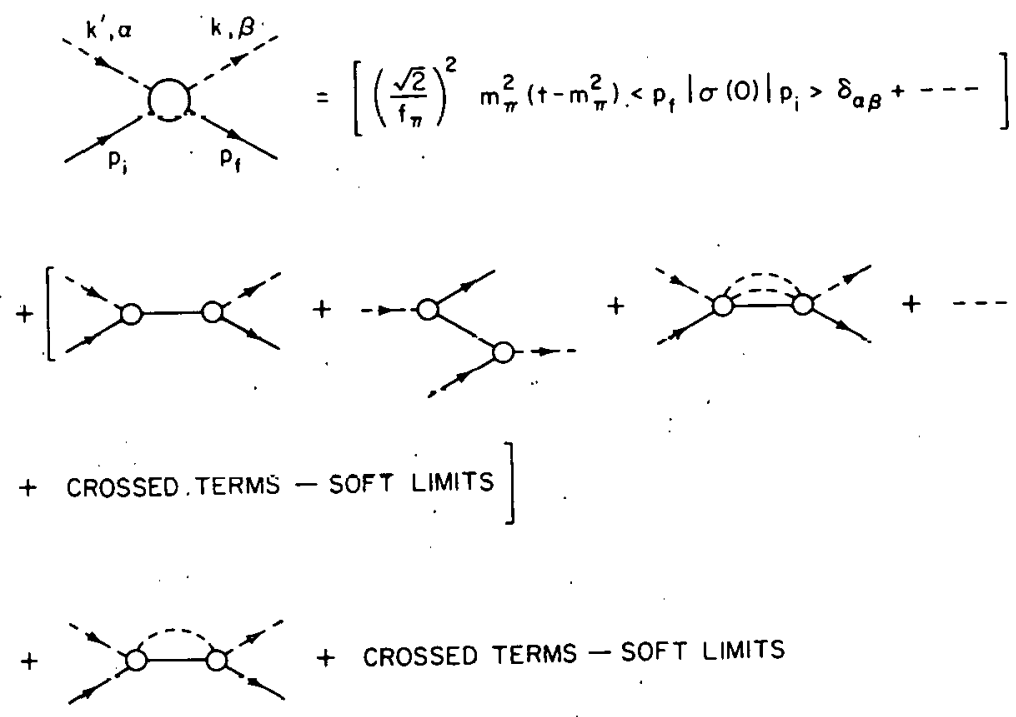

Fig. 1: Once-subtracted Low expansion. 
The analogy between this integral equation and Eq. (1) is transparent. The first group of terms on the right-hand side make up the soft pion limit. Since pions can be absorbed and emitted and can dissociate into $\mathrm{NN}$ pair we get the middle group of terms. The last group involve integrals over the $\pi N$ scattering amplitudes and their complex conjugates. The first two groups are the driving terms for the integral equation. In the work on low energy $S$ wave phase shifts that we did two years back we did not include $S$ wave inelasticity.7,10 The nucleon pole terms have only one unknown ingredient, namely, the $\pi N$ form factor for $t<0$. Note that our assumption of PCAC fixes $g_{\pi}(0)=\frac{\sqrt{2} \mathrm{Mm}_{\pi}^{2}}{f_{\pi}} g_{A}(0)$ which is the GoldbergerTreiman ${ }^{11}$ relation. The nucleon pole terms play a relatively minor role and so we do not determine the form factor for $t<0$ very well.

Our main driving terms are the $\sigma$ commutator term, the main isoscalar mechanism, and the Z-graphs, the main isovector mechanism. The subtraction of the soft limits kills the huge isocalar part of the 2-graphs, thus gimulating pair suppression.

Evaluation of the $z$-graphs require the quantity $g_{\pi}\left(t \geq 4 M^{2}\right)$. At present it is not practical to calculate its value reliably. However, the various studies 12 of $\pi N$ form factor assure us that $\mathrm{g}_{\pi}\left(t \geq 4 \mathrm{M}^{2}\right)$ is not likely to be small compared to $g_{\pi}(0)$. Through a slight approximation we arrange matters such that we need to know on $1 y \cdot g_{\pi}\left(t \geq 4 \dot{M}^{2}\right) \mid$ which we parametrize as

$$
\left|g_{\pi}\left(t \geq 4 \dot{M}^{2}\right)\right|=\frac{\bar{g}_{\pi}=\left|g_{\pi}\left(4 M^{2}\right)\right|}{1+\frac{L=4 M^{2}}{4 m_{0}^{2}}} .
$$

We parametrized the $\pi$ commutator matrix oloment ao

$$
\left.{ }^{<p_{f}}|\sigma(0)| p_{i}\right\rangle=\frac{\sigma(\pi N)}{\left(1-\frac{t}{m_{1}^{2}}\right)\left(1-\frac{t}{m_{2}^{2}}\right)^{2}} \text {. }
$$

Commonly the quantity $\sigma(\pi N)$ is referred to as the $\sigma$ commutator.

Our $S$ wave equations have another type of driving term which is not manifest in the pictorial representation. Our choice of the CM frame guarantees that the $\pi N$ system in the right-hand cut is at rest. But the $\pi N$ system is not at rest in the crossed term nor in the soft pion limits. Because of this the $P$ wave (and, in principle, all higher waves) amplitudes do provide a small but nonnegligible effect. The resulting driving term is parametrized in terms of the $P$ wave cross sections and form factors by making a separability ansatz. The form factor is assumed to be of the form $7,10 \mathrm{k} /\left(1+\mathrm{k}^{2} / \mathrm{\mu}^{2}\right)^{5 / 2}$.

Naturally, our low energy $S$ wave work cannot fix the various form factors very precisely. In Refs. 7 and 10 we quoted the values $\mathrm{m}_{0}=8.6 \mathrm{~m}_{\pi}, \mathrm{m}_{1}=8.24 \mathrm{~m}_{\pi}, \mathrm{m}_{2}=7.5 \mathrm{~m}_{\pi}$ and $\mu=8 \mathrm{~m}_{\pi}$. But for any given set of values of the form factor masses the sensitivity of the $S$ wave phase shifts on the main parameters $\pi(\pi N)$ and $\overline{\mathbf{g}}_{\pi} 1 s$ very marked. In Fig. 2 we exhibit experimental and theoretical phase shifts. The solid lines are the energy dependent fits of 


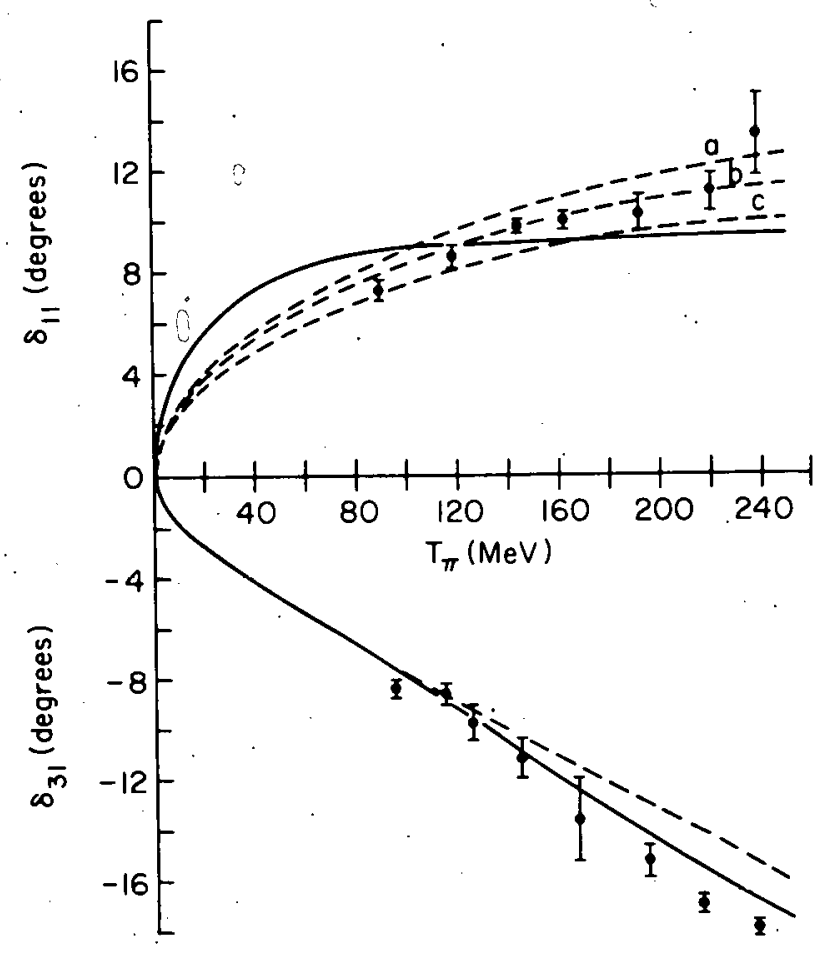

Fig. 2: Experimental and theoretical $\mathrm{S}$ wave phase shifts.
Zideil, Roper and Arndt; 13 the flagged points are the energy independent fit phase shifts of Carter, Bugg and Carter. 14 The dashed lines are theoretical calculations for three sets of $\sigma(\pi N)$ and $\bar{g}_{\pi}$, listed below.

\begin{tabular}{|c|c|c|}
\hline Curve & $\sigma(\pi N)$ & $\overline{\mathrm{g}}$ \\
\hline ? & $0 \mathrm{MeV}$ & \\
\hline b & $5 \mathrm{MeV}$ & \\
\hline c & $26.0 \mathrm{MeV}$ & 1 \\
\hline
\end{tabular}

The values were chosen such that they gave the same $\delta_{31}$. We draw two conclusions. First, the energy dependent fits are unacceptable at low energies. It is nearly impossible to reproduce theoretically the sharp change of slope of $\delta_{11}$. Second, as long as the true phase shifts are close to those of Carter, Bugg and Carter, the parameters $\sigma(\pi N)$ and $\bar{\varepsilon}_{\bar{\pi}}$ will have values slose to the ones shown horo. We obtained the following scattering lengths, $a^{(+)}=\frac{1}{2}\left[a\left(\pi^{-} p\right)+a\left(\pi^{+} p\right)\right]=$ $-0.016 \mathrm{~m}_{\pi}^{-1}$ and $\mathrm{a}^{(-)}=\frac{1}{2}\left[\mathrm{a}\left(\pi^{-} \mathrm{p}\right)-\mathrm{a}\left(\pi^{+} \mathrm{p}\right)\right]=0.079 \mathrm{~m}_{\pi}^{-1}$, which agrees exactly with the current algebra prediction of Weinberg. 15

Current algebra provides another check of our theoretical work in the form of the following sum rule, 7

$$
\left(\frac{\sqrt{2}}{f_{\pi}}\right) 2 \frac{m_{\pi}^{4}}{2}=\frac{1}{2 M^{2}}\left|g_{\pi}\left(4 M^{2}\right)\right|^{2}+\frac{M}{6 \pi^{2}} \int \frac{q^{2} d q}{\omega(q) E(q)} \frac{\left|f_{11}(q, 0)\right|^{2}-\left|f_{3 I}(q, 0)\right|^{2}}{[E(q)+\omega(q)-M]^{2}} \text {, }
$$

where $f_{2 I, 2 J}(q, 0)$ is a half-off-mass shell $S$ wave scattering amplitude. We'satisfy the sum rule within the numerical accuracy of our work. The sum rule may also be interpreted as providing a check on our value for $\bar{g}_{\pi}$.

Our value of $\sigma(\pi N) \sim 25 \mathrm{MeV}$ is in sharp disagreement with the value of $\sim 65 \mathrm{MeV}$ obtained in recent years by various authors using dispersion relations method.16,17,18 But significantly our value is close to those obtained in the 1ate 1960's 19 using the FubiniFurlan theory. 20 The dispersion relations method is based on an Idea proposed by Osypowsk121 and by Cheng and Dashen. 22 It deals with the amplitude 


$$
\bar{C}^{(t)}(v, t)=A^{(t)}(v, t)+\frac{j v}{1-\frac{t}{4 M^{2}}} B^{(t)}(v, t)-\frac{G_{\pi}^{2}}{M\left(1-\frac{t}{4 M^{2}}\right)} \frac{v_{B}^{2}}{v_{B}^{2}-v^{2}}
$$

where $A$ and $B$ are the usual $C_{G L N}^{2}$ amplitudes, with pions and nucleons in their mass shel1. $v=(s-u) / 4 M, v_{B}=\left(t-2 m_{\pi}^{2}\right) / 4 M$ and $G_{\pi}=g_{\pi}\left(m_{\pi}^{2}\right)$ is the $\pi N$ coupling constant. The authors observed that

$$
\overline{\mathrm{C}}^{(+)}\left(0,2 \mathrm{~m}_{\pi}^{2}\right)=\left(\frac{\sqrt{2} \mathrm{~m}_{\pi}^{2}}{\mathrm{f}}\right)^{2} \sigma(\pi \mathrm{N})-\frac{\mathrm{m}_{\pi}^{2} \mathrm{G}}{2 \mathrm{M}^{3}}+O\left(\mathrm{~m}_{\pi}^{4}\right) \ldots
$$

The left-hand side is an on-mass-shell amplitude for unphysical energy and momentum transfer and, therefore, can be determined from dispersion relations. The authors conjectured that the terms $\sim O\left(\mathrm{~m}_{\pi}^{4}\right)$ are negligible and so the above relation provides a method of determining $\sigma(\pi \mathrm{N})$. In practice there are three steps involved. First, one must determine a set of amplitudes $\bar{C}( \pm)\left(\nu>m_{\pi}, t \leq 0\right)$ and $B( \pm)(\nu>$. $m_{\pi}, t \leq 0$ ) for physical values of $v$ and $t$ using fixed $t$ dispersion relations in the variable $\nu .23$ It is in the course of this that one also determines the coupling constant $\mathrm{f}^{2}=\frac{\mathrm{G}_{\pi}^{2}}{4 \pi} \frac{\mathrm{m}_{\pi}^{2}}{4 \mathrm{M}^{2}}$. In the second step one determines the values of $\bar{C}^{(+)}(0, t<0)$. Finally $\bar{C}^{(+)}\left(0,2 \mathrm{~m}_{\pi}^{2}\right)$ is evaluated with some extrapolation technique.

The amplitudes $\overline{\mathrm{C}}(+)(0, t<0)$ involve pure imaginary space momentum $\sqrt{\left(\frac{t^{2}}{16}-M^{2} m_{\pi}^{2}\right) /\left(M^{2}+m_{\pi}^{2}-\frac{t}{2}\right)}$ for small $t$. But we noted that as long as the magnitude of the space momentum is small it is in the domain of analyticity of the scattering amplitude as a function of the momenta. Thus we could use our theory to determine $\bar{C}(t)(0, t<0)$ for small t.

The quantity $O\left(\mathrm{~m}_{\pi}^{4}\right)$ in Eq. (2) can be determined only with a dynamical thcory. Wc cvaluated it and found it to contribute $\sim 4 \mathrm{MeV}$ to $\sigma(\pi \mathrm{N})$. While the error is small compared to the discrepancy between $25 \mathrm{MeV}$ and $65 \mathrm{MeV}$ it is totally unacceptable for a phase shift calculation.

In. Fig. 3 we present $\overline{\mathrm{C}}^{(+)}(0, t<0)$ s determined by Langbcin ${ }^{17}$ as illustrative of typical dispersion relation method calculations. The solid curves are our theoretical calculations. 24 The curve a is based on parameters quoted in our paper on $S$ wave phase shifts. For a theoretical calculation, with all parameters fixed a priori, the agreement for small $t$ is most encouraging. We then found that we can noticeably improve the $f$ it by changing the $P$ wave form factor mass $\mu$ from $8 m_{\pi}$ to $10 m_{\pi}$. To get the same low energy $s$ wave phase shifts it was necessary to increase $\bar{g}_{\pi}$ to 11.9 and decrease $o(\pi N)$ to $24.9 \mathrm{MeV}$. The curve $b$ is due to this set of parameters.

In view of the nice agreement for small $t$ we are inevitably led to the conclusion that there. is no real discrepancy between our value and the results of the dispersion relation methods. While the art of determining the best set of amplitudes from dispersion relation is at a very high level the art of determining the error bars on these amplitudes is far behind. The errors on $\sigma(\pi \mathrm{N})$ 


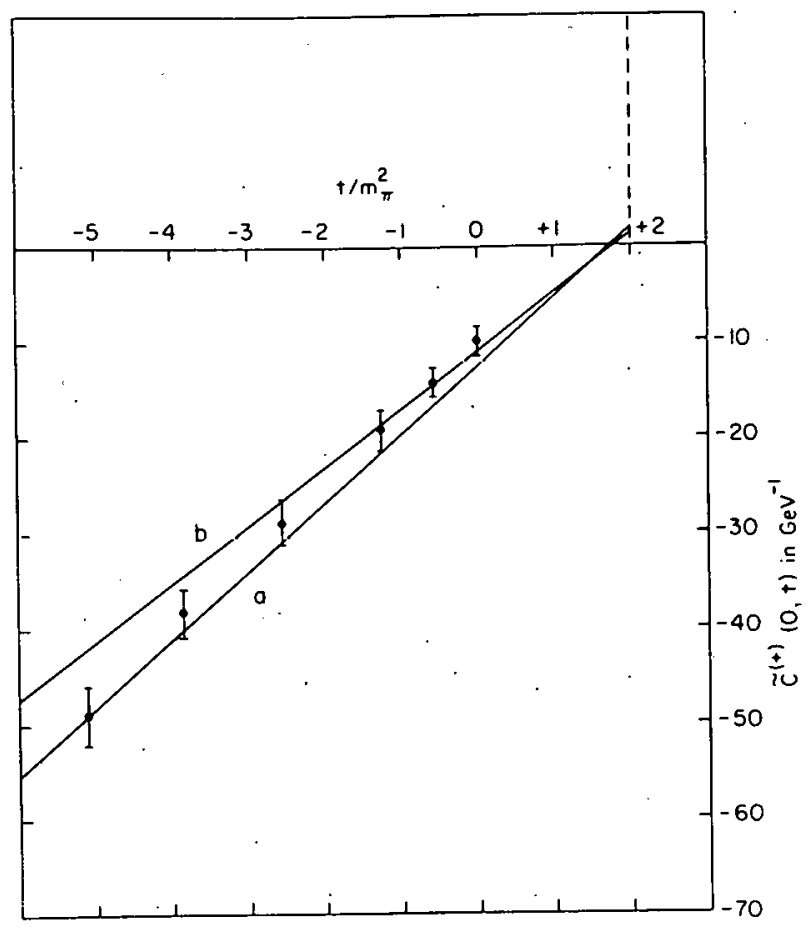

Fig. 3: $\overline{\mathrm{C}}^{(+)}(0, t)$ amplitudes. quoted in many papers are simply not based on careful analysis of the problem. For the evaluation of $\sigma(\pi N)$ considerable care must be exercisped as the amplitude $\overline{\mathrm{C}}(\mathrm{f}) \mathrm{(0,t)}$ has a node very close to $t=2 m_{\pi}^{2}$. We should also point out that any theory of $\pi \mathrm{N}$ interaction which claims to be reasonable should be capable of yielding values of $\bar{c}^{(+)}(0, t)$ for small $t$. In fact these amplitudes are of the "off-energy shell" category a la Blancenbecler and Sugar. 25 Thus dispersion relations provide a check on models of offenergy shell $\pi \mathrm{N}$ amplitudes. A parallel situation does not exist for NN amplitudes.

Before moving of $f$ this subject we may note that the $\sigma$ model coupled with the notion of pair suppression gives $\sigma(\pi \mathrm{N}) \sim 26 \mathrm{MeV}$. According to the $\sigma$ model the $\sigma$ commutator $\sigma=\sigma_{\text {field }} \frac{\hat{f}_{\pi}}{\sqrt{2}}$ Thus $\sigma(\pi N)=\frac{\mathrm{f}_{\pi}}{\sqrt{2}} \frac{g_{\sigma N N}}{m_{\sigma}^{2}}$. But pair suppression requires $\mathrm{g}_{\sigma \mathrm{NN}} / \mathrm{m}_{\sigma}^{2}=\mathrm{g}_{\pi} / \mathrm{M}^{2}$. Combining the two and using Goldberger-Treiman relation we have $\sigma(\pi \mathrm{N})=\frac{\mathrm{m}_{\pi}^{2} \mathrm{~g}_{\Lambda}(0)}{\mathrm{M}}=26 \mathrm{MeV}$.

Up until now we have tested our theoretical amplitudes for small values of momenta only. It is possible that our successes have been spurious. Another source of worry is the value of $\mathrm{f}^{2}=\frac{\mathrm{G}^{2}}{4} \frac{\mathrm{m}^{2}}{4 \mathrm{M}^{2}}$ which according to dispersion theorists is $0.079 \pm 0.001 .26$ As PCAC is the central assumption of our theory we are locked into a value $\sim 0,0725$ which includes a $3 \%$ enhancement due to form factor. However, there is a class of tests, to which we can subject our theory, which touches on both these issues.

Consider the unsubtracted forward dispersion relation

$$
\frac{M+m_{\pi}}{M m_{\pi}} a^{(-)}-\frac{2}{m_{\pi}^{2}} \frac{f^{2}}{1-\frac{m_{\pi}^{2}}{4 M^{2}}}=J=\frac{1}{2 \pi^{2}} \int \frac{d k}{\omega} \sigma^{(-)}(\omega) \ldots
$$

and the once-subtracted relations 


$$
\begin{aligned}
& \frac{4 \pi\left(M+m_{\pi}\right)}{M m} a^{(-)}-\bar{c}^{(-)}(0,0)-\frac{8 \pi f^{2}}{M\left(1-\frac{\pi}{4 M^{2}}\right)}=I(-)=\frac{2 m^{2}}{\pi} \int \frac{d k}{\omega^{3}} \sigma^{(-)}(\omega) \\
& \frac{4 \pi\left(M+m_{\pi}\right)}{M} a^{(+)}-\bar{c}^{(+)}(0,0)+\frac{4 \pi f^{2}}{m^{2}}=m_{\pi} I^{(+)}=\frac{2 m^{2}}{\pi} \int \frac{d k}{\omega^{2}} \sigma^{(+)}(\omega) .
\end{aligned}
$$

It is useful to remind ourselves that $f^{2}$ is determined in the course of evaluation of the amplitudes $\bar{C}( \pm)(\nu, t)$ and $B( \pm)(\nu, t)$ using fixed $t$ dispersion relations $g$ which the above are special cases. Also, we may note that if a ${ }^{(-)}$was directly measurable one could use Eq. (3) to determine $f^{2}$. Unfortunately, a $(-)$ is not directly measurable.

From our low energy theory we can calculate $27 a^{( \pm)}$and $\bar{C}( \pm)(0,0)$. As explained before $\mathrm{f}^{2}=0.0725$ is practically a fixed parameter of the theory. Thus we have predictions for the cross section integrals via dispersion relations. We can then test our theory by comparing our results with those obtained by the dispersion relation methods. In Table I we present our theoretical results for parameter set b, described earlier and the results obtained by Höhler, Koch and Pietarinen. 18

TABLE I

\begin{tabular}{ccc}
\hline Integral & Ours $\left(\mathrm{m}_{\pi}^{-2}\right)$ & Höhler, et al. $\left(\mathrm{m}_{\pi}^{-2}\right)$ \\
\hline $\mathrm{J}$ & -0.056 & -0.053 \\
$\mathrm{I}^{(-)}$ & -0.202 & -0.206 \\
$\mathrm{I}^{(+)}$ & -1.39 & 1.47 \\
\hline
\end{tabular}

We consider the agreement eminently pleasing.

Work on the $P$ wave is still in progress. 28 one of the things we have learnt is that the dynamical picture which emerges from a static theory is not the whole story. Details will be presented in a contributed paper.

\section{REFERENCES}

1. G. F. Chew and F. E. Low, Phys, Rev, 101, 1570 (1956).

2. For example, G. F. Chew, M. L. Goldberger, F. E. Low and Y. Nambu, Phys. Rev. 106, 1337 (1957).

3. For a review see Current Algebras by S. L. Adler and R. F. Dashen (Benjamin, N. Y:, 1968).

4. For example, R. Landau and F. Tabakin, Phys. Rev. D5, 2746 (1972).

5. For example, D. J. Ernst and M. Johnson in Meson-Nuclear Physics1976, proceedings of the International Topical Conference.

6. J. B. Cammarata and M. K. Banerjee, Meson-Nuclear Physics-1976, proceedings of the International Toplcal Conference.

7. M. K. Banerjee and J. B. Cammarata, Phys. Rev. D16, 1334 (1977). 
8. M. Gel1-Mann and M. Levy, Nuovo Cimento 16, 705 (1960).

9. J. L. Basdevant and B. W. Lee, Phys. Rev. D2, 1680 (1970).

10. For technical details see M. K. Banerjee and J. B. Cammarata, Phys. Rev. C17, 1125 (1978).

11. M. L. Goldberger and S. B. Treiman, Phys. Rev. 110, 1178 (1958).

12. H. F. Jones and M. D. Scadron, Phys. Rev. Dl1, $\overline{174}$ (1875); H. Pagels and A. Zepeda, Phys. Rev. D5, 3262 (1972); J. B. Cammarata and M. K. Banerjee, Bull. Am. Phys. Soc. 20, 1192 (1975); W. Nutt and B. Loiseau, Nucl. Phys. B104, 98 (1976).

13. V. S. Zidell, L. D. Roper and R. A. Arndt, private communication.

14. J. R. Carter, D. V. Bugg and A. A. Carter, Nucl. Phys. B58, 378 (1973).

15. S. Weinberg, Phys. Rev. Lett. 17,616 (1966).

16. H. Nielson and G. C. Oades, Nucl. Phys. B72, 310 (1974).

17. W. Langbein, Nuc1. Phys. B94, 519 (1975).

18. G. Höhler, R. Koch and E. Pletarinen, Karlsruhe Report No. TKP77-11 (unpub1ished).

19. F. Von Hippel and J. K. Kim, Phys. Rev. D1, 151 (1970); 3 , 2923(E) (1970); W. T. Huang, C. A. Levinson and M. K. Bañerjee, Phys. Rev. C5, 651 (1972).

20. S. Fubini and G. Furlan, Ann. Phys. (N.Y.) 48, 322 (1968).

21. E. T. Osypowsk1, Nuc1. Phys. B21, $615(1970)$.

22. T. P. Cheng and R. Dashen, Phys. Rev. Lett. 26, 594 (1971).

23. For example, E. Pietarinen, Nuc1. Phys. B107, 21 (1976).

24. M. K. Banerjee and J. B. Cammarata, Phys. Rev. D18, 4078 (1978).

25. R. Blankenbecler and R. Sugar, Phys. Rev. 142, $\overline{1051}$ (1966).

26. G. E. Hite, R. J. Jacob and D. C. Joir, Phys. Rev. D12, 2677 (1975).

27. M. K. Banerjee and J. B. Cammarata, Phys. Rev. D19, 145 (1979).

28. In collaboration with Nien-Chih Wei. 